\title{
Feedback Implosion Suppression Algorithm for Reliable Multicast Data Transmission over Geostationary Satellite Networks
}

\author{
A.Sali, G.Acar, B.Evans \\ Centre for Communication Systems Research, \\ University of Surrey, \\ Guildford, GU2 7XH, United Kingdom \\ \{A.Sali, G.Acar, B.Evans\}@ surrey.ac.uk
}

\author{
G.Giambene \\ CNIT-Università degli Studi di Siena, \\ Via Roma, 56, \\ 53100 Siena, Italy \\ giambene $a$ unisi.it
}

\begin{abstract}
In this paper, we propose a feedback implosion suppression (FIS) algorithm that reduces the volume of feedback traffic from receivers in a multicast group over a geostationary satellite network. The system considered in this paper includes a reliable multicast transport protocol that operates on top of a channel-aware scheduler (CAS) with receivers capable of measuring and feeding back to the scheduler their channel state information (CSI). The collected CSI becomes a valuable input to avoid unfavorable channel conditions to reduce the forward link resources that would be wasted to retransmit lost segments. Using a fixed number of available uplink slots, the Radio Network Controller (RNC) decides which receivers to update their feedback messages in the next collection period based on their CSI in the current cycle. The integration of FIS and CAS modules achieves a degree of full reliability in multicast transmission over a satellite network without relying on any collaboration between receivers, or on any infrastructure other than the satellite network.
\end{abstract}

Keywords- feedback implosion suppression, reliable transmission, multicast, satellite communication.

\section{INTRODUCTION}

Research on cross-layer design has recently attracted significant interest. Cross-layer design suggests (possibly joint) adaptation of communication protocols and mechanisms at various layers according to the information collected at other layers of the communication system. Recent growth in heterogeneous networks entails adaptive mechanisms. In this framework, a cross layer approach would be more effective and flexible. In wireless systems where both radio resources and power are strictly constrained, resource optimization is needed when such opportunity is not guaranteed by the current layered protocol stack.

In the case of interaction between physical and MAC layers, the user feedback would be a valuable input to various components in the MAC layer to achieve a degree of reliability in transmission of multicast data in GEO satellite network. A CSI collection policy is proposed in [1] where CSI from the physical layer is updated and exploited by the scheduler at the MAC layer. The periodic CSI updates become the input to the scheduler to make a decision to transmit a particular segment in the current timeslot. The scheduling algorithm delays the segment until the probability of retransmission for the segment is less than a specified upperbound value. The paper shows that the scheduling policy improves the total session delay by reducing the number of segments retransmitted and by exploitation of reported CSI values.

In [1], CSI updates are expected periodically from all active receivers in the multicast group. This may create feedback implosion problem as the receivers try to send their feedback messages through the return link. In satellite-based networks, this phenomenon could result in a holding up of uplink resources and cause congestion. Thus, there exists a need for a suitable FIS policy in this context to complement the CSI collection policy as proposed in [2]. In [2], the authors present how feedback implosion problem is tackled by a receiverselection policy onto a fixed number of available uplink slots. However, the percentage of suppressed receivers due to collision is still high although a collision avoidance strategy has been proposed, causing high average error between the CSI collected using FIS at the RNC, and the CSI measured at the user's terminal.

Another interesting possible implementation of reliable multicast over geo-satellite networks can be found in [3], where the return link between the receivers and the satellite to communicate retransmission request are conveyed via High Altitude Platforms (HAPs). Although the costly nature of satellite bandwidth and the high transmission power in the uplink is avoided, the approach obviously assumes that receivers are connected to HAP. However, our philosophy in this paper is to study reliable multicast transmission in a satellite deployment without any collaboration with any terrestrial network infrastructure.

In this paper, we investigate the possibility of reducing the volume of receivers' feedback traffic by exploiting the nature of CSI by considering that the CSI values may contain redundant information due to correlation of channel variations and the fact that the protocol may only need to track the behavior of a subset of receivers with large channel variation. Our work significantly differs from [2] in two ways (i) collision problem is avoided altogether and, (ii) we include a "update age" parameter for fair treatment of receivers that 
have been suppressed for long intervals and for increased robustness.

In the next section, we present the satellite network architecture. In Section III, the FIS policy is described, and in Section IV the integration of FIS and channel-aware scheduling algorithm is explained. In Section $\mathrm{V}$ a comparable policy is investigated; namely CSI updates using random access mechanism, and finally we conclude the paper in Section VI.

\section{SYSTEM DESCRIPTION}

The reference system is based on an L-Band geostationary satellite network similar to Inmarsat BGAN (Broadband Global Area Network) system. The scheduling and feedback implosion suppression mechanism is executed at the Radio Network Controller (RNC) and a geostationary satellite relays multicast data product to all multicast receivers through Mobile Terminals (MT) and Terminal Equipment (TE). Currently, we adopt a single satellite and single spotbeam scenario. We assume an $N$ number of active receivers, possibly, experiencing different propagation conditions. The receivers are set to experience a percentage of simulation time in one propagation environment type before moving to another propagation type to simulate time-varying channel property. In this paper, we use an L-band channel model based on propagation parameters from [4]. The propagation parameters are recommended values to match the extracted time-series parameters from measurement. The channel model is based on the specific propagation environments namely suburban and wooded areas with different elevation angles and channel conditions.

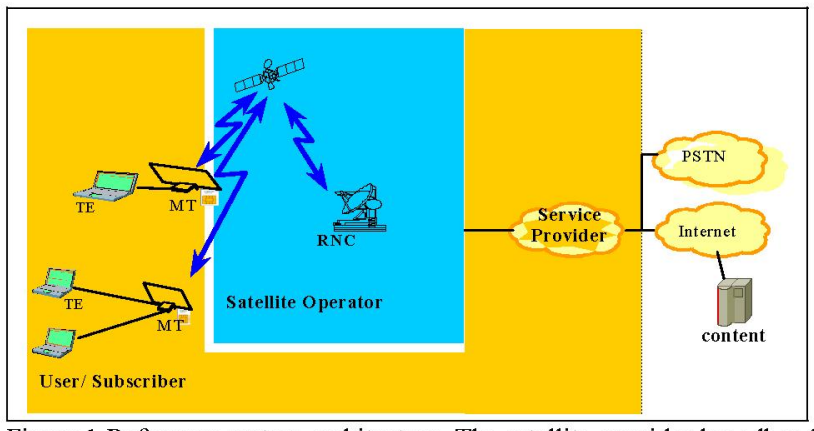

Figure 1 Reference system architecture. The satellite provides broadband access for transmission of multicast data product to receivers.

We assume that multicast reliability is achieved via a multicast transport protocol with a retransmission strategy that is based on StarBurst MFTP (Multicast File Transfer Protocol) [5]. More specifically, via this higher-layer protocol, we assume that a multicast file is transmitted to all group members in its entirety. In response, the receivers send negative acknowledgments (NACK) indicating lost segments. The receivers acquire access by using limited dedicated uplink slots, which invokes the FIS algorithm to share the uplink capacity among all active receivers.

III. FEEDBACK IMPLOSION SUPPRESSION ALGORITHM
The CSI collection protocol performs between the RNC and the group of direct receivers located inside a spotbeam in an attempt to collect periodic CSI and ACK/NACK semantics. Receivers submit their feedback information by accessing the uplink channel and sending a message feedback. It is assumed that a feedback message from one user can be transmitted in a single uplink slot. The resource allocation algorithm shares the available uplink slots among all active receivers based on the feedback from each receiver. The result of the resource allocation is broadcast to all active receivers.

The CSI of interest in our case is packet error rate, $P$, for multicast receivers, denoted with index $j$ from 1 to $N$. The goal of the scheduling algorithm is to calculate the probability of retransmission for block $i$ at every collection period $n$, $P_{R t x}^{i}(n)$, using the feedback received from all active receivers in a group. In order to reduce the volume of feedback information that is sent through the network, the FIS algorithm modifies the CSI collection policy by collecting feedback information from a subset of receivers $\breve{N}$ out of all active receivers $N$, using a fixed amount of available uplink slots, $S_{n}$. Therefore, not all feedback information state variables are updated at every collection period.

Assuming that the set of all active receivers $N$ are available at $\mathrm{RNC}$ at all times, the protocol updates state variables and calculates a new probability of retransmission for block $i$ at every collection period $n, P_{R t x}^{i}(n)$ by a two-step process:

1) Let $N$ denote the set of active receivers at the start of collection period $n$ at time $t=n T$, where $T$, is the collection period, and let $\mathrm{P}_{j}(n T)$ denote the packet error rate, sampled by user $u_{j}$ at the collection period. Receivers send their measurements to the RNC, which collects and updates the values:

$$
\hat{P}_{j}[n] \leftarrow P_{j}(n T) \forall j \in N
$$

2) After the collection, the RNC calculates the new probability of retransmission

$$
\hat{P}_{R t x}^{i}[n]=1-\prod_{j=1}^{N}\left(1-\hat{P}_{j}[n]\right)
$$

The update operation in (1) requires all active receivers to acquire access to uplink slots to transmit their feedback message. The feedback volume would be minimized if only a subset of receivers responded at every collection period. Thus, the feedback implosion suppression policy modifies the behavior of CSI collection policy such that only a subset of active receivers, report their CSI measurement using $S_{n}$ uplink slots for the collection period. Therefore, the state variables $P_{j}$ are updated at the end of collection period $n$ as:

$$
\begin{aligned}
& \hat{P}_{j}[n] \leftarrow P_{j}(n T) \forall j \in \breve{N} \\
& \hat{P}_{j}[n] \leftarrow P_{j}[n-1] \forall j \notin \breve{N}
\end{aligned}
$$


The probability of retransmission calculated using the state variables may not be equal to the probability of retransmission measured at time $t=n T$. In other words, the receivers which are selected to access the uplink slots and update their CSI values in the current collection period may experience a different value of CSI due to round trip propagation delay. Therefore, there exists discrepancy between $\hat{P}_{R t x}^{i}[n]$ which is calculated at the RNC using the FIS algorithm, and $P_{R t x}^{i}(t)$ measured at time $t=n T$. In essence, error could occur due to this discrepancy, which is from (i) FIS formulation, and (ii) round trip propagation delay. This error may impact the channelaware transmission in terms of multicast protocol parameters, which will be analyzed in performance evaluation.

The system may reserve a fixed number of uplink slots for feedback transmission, or the value of $S_{n}$ may be determined by the remaining slots after user requests for data transmission are accommodated. The FIS policy selects receivers and assigns them to one of the $S_{n}$ available uplink slots is a knapsack problem and closely represented by multiple subset sum problem (MSSP) formulation [6]. Mathematically, the MSSP can be described as follows:

Definition 1: Given a set $\left\{u_{j}\right\}_{j=1}^{N}$ of items, each item has a positive weight $r_{j}$, and a set of $\left\{b_{i}\right\}_{i=1}^{S_{n}}$ of identical bins each having a positive capacity $R_{\text {Max }}$, what is the assignment that maximizes the:

$$
\begin{gathered}
\max \sum_{i=1}^{S_{n}} \sum_{j=1}^{N} r_{j} x_{j i} \\
\text { subject to } \sum_{j=1}^{N} r_{j} x_{j i} \leq R_{M a x}, \forall i
\end{gathered}
$$

Heuristically, the assignment aims to optimize the selection and the assignment to active receivers of uplink slots by selecting only $\breve{N}$ number of active receivers to access the $S_{n}$ available uplink slots. The FIS protocol solves an instance of MSSP with:

$$
r_{j}^{\prime}=\frac{\omega_{j}}{\phi_{j}} \times \sqrt{\Delta t_{\text {Update }, j}}
$$

where $\omega_{j}=\min \left\{\left|\theta_{1}^{j}-\theta_{0}^{j}\right|, \omega_{\text {Max }}\right\}$, and $\Delta t_{\text {Update }, j}=\min \left\{\left(n-n_{\text {last }}^{j}\right), \Delta t_{\text {Max }}\right\}$.

The indication of ACK/NACK semantics are represented by $\phi_{j}$ which takes the value of $\phi_{j}=2$ if the block $i$ is correctly received ( $\mathrm{ACK}$ ) and $\phi_{j}=1$ if at least one of the segments $p$ in the block $i$ is in error (NACK). The age of a feedback sample is represented by a term called $\Delta t_{U p d a t e, j}$ denoting the difference of time between the last CSI update and the current collection period. The parameter $\omega_{j}$ represents the difference between current CSI value and its past value. A set of upperbound values are imposed for $\omega_{j}$ and $\Delta t_{U p d a t e, j}$ such that any value above the maximum level is treated equally. In essence, in this formulation, a user $u_{j}$ with high $r_{j}$ is favored over other receivers with lower $r_{j}$.

Let $\mathbf{x}^{\mathrm{n}}$ be the solution vector that represents the assignment of items to bins. This represents the assignment of receivers to uplink slots, such that $x_{j}^{n}=1$ if user $u_{j}$ is selected and assigned to slot $i=\left\{1,2, \ldots, S_{n}\right\}$. From (5), there will exist a situation when more than one user is allocated to one slot. In this tiebreaking case, the RNC decides that the slot is allocated to the user with the highest $\Delta t_{\text {Update }, j}$ so that a user with a previously lower $r_{j}$ but currently having a higher value of $\Delta t_{\text {Update }}$ still have the opportunity to be selected. This approach increases adaptivity of the FIS algorithm against changes in the channel condition. In general, the FIS gives priority to receivers with large variance of CSI values, receivers who have been suppressed longer and receivers with NACK. The RNC broadcasts the solution vector $\mathbf{x}^{\mathrm{n}}$ over the network as part of the bandwidth allocation procedure and reserves the necessary uplink slots for the collection period. The set $\breve{N}$ is constructed as:

$$
\breve{N}=\left\{u_{j}: x_{j}^{n}=1\right\}
$$

which represents the selected receivers and the value of $\breve{N}$ is equal to $S_{n}$.

\section{INTEGRATION OF FEEDBACK IMPLOSION SUPPRESSION INTO CHANNEL-AW ARE SCHEDULER (FIS+CAS)}

In this section we describe how the proposed feedback implosion suppression works with the channel-aware scheduler to provide reliable transmission of multicast data over satellite networks. We assume the data is segmented into $B$ blocks, and each block $i$ has $D$ number of segments. Transmission of batches proceeds in transmission rounds. In transmission round $k=\{1,2, \ldots, k M a x)$, the protocol attempts transmission of $r(k)$ number of blocks. However, in the initial transmission round $k=0$, the number of blocks is equal to the total number of blocks in the file: $r(0)=B$. Upon reception of transmitted blocks, receivers note which segments are corrupted in each block. Then, each receiver sends a feedback containing bitmap representing NACK semantics of segment $p$ in the received block $i ; \phi(p, i)=\{1,2\}$ : a value of ' 1 ', representing NACK and ' 2 ' representing $A C K$. At the RNC, the maximum number of segments, $r$, needs to be transmitted in the next transmission pass $(k+1)$, is calculated using the feedback messages:

$$
r(k+1)=\sum_{i=1}^{B} A_{i}(k)
$$

where $A_{i}(k)$ is the number of segments required to be retransmitted from block $i=\{1,2, \ldots, B\}$ with initially $A_{i}(0)=0$. $A_{i}(k)$ is calculated over all receivers as: 


$$
A_{i}(k)=\max _{j \in N}\left\{A_{i}^{j}(k)\right\}
$$

The protocol then proceeds with the next transmission pass until all blocks are completed successfully by all receivers. An average error is expected as there exists a difference between the value of probability of retransmission with FIS calculated at the RNC, and the value of probability of retransmission calculated at the receivers' terminals. In other words, we observe the error due to the FIS algorithm in place and also due to round trip propagation delay. Note that the selection and allocation decision in FIS is based on feedback information successfully reported to the RNC, rather than the current CSI values measured by the user. Hence for a given number of uplink slots, the average error increases as the number of active receivers increase. This is expected as the algorithm selects and allocates receivers from a larger set to a fix number of slots. Therefore, more receivers are suppressed by the algorithm giving less accurate calculation of probability of retransmission. However, from a system provider's or receivers' point of view, the important performance metric would be the effect of the error introduced by the FIS algorithm to the scheduling and transmission mechanism.

V. CSI Updates USINg RANDOM ACCESs Mechanism

A random access mechanism to access the uplink slots is compared with our integrated FIS and channel-aware scheduler (FIS+CAS) policy. In the random access mechanism the transport protocol is chosen to be MFTP-like because it provides a considerable degree of reliability in transmission of large files and hence comparable with our proposal. We refer this random access mechanism as MFTP+CSI policy. In this policy, a user with NACK semantics after the end of a block reception shall acquire a reservation slot to update its CSI values using the contention slots.

Correspondingly, the value of $\hat{P}_{R t x}[n]$ is calculated using (2) from the reported $P_{j}$ according to:

$$
\hat{P}_{j}[n]=\left\{\begin{array}{l}
P_{j}(n T), j \in N_{\phi} \\
P_{j}[n-1], j \notin N_{\phi}
\end{array}\right.
$$

where $P_{j}(n T)$ is packet error rate measured at the user terminal and successfully updated at the $\mathrm{RNC}, P_{j}[n-1]$ is packet error rate from the last update of a particular user $j$ and $N_{\phi}$ is total number of receivers successfully access the contention slot and occupy the reservation slot in the uplink.

For comparison, note that in FIS + CAS, $\breve{N}=S_{n}$ since one feedback message triggered by a user is encapsulated within one uplink slot. However, $\breve{N} \neq N_{\phi}$ because in any particular collection period, the value of $N_{\phi}$ can be any integer from 0 to the highest number of contention slots, considering some feedback messages is lost due to collision. Although feedback messages experiencing collision will subsequently be retransmitted, but a scenario will occur when the retransmission is successfully received at the RNC only after the StatusRetryTimer expires. In this case that particular feedback message will be considered in the next poll. In this note, a CSI value from any particular user will not be updated at the RNC in the current collection period due to: ( $i$ ) the user do not access the uplink slot because all segments are successfully received at the user's terminal, or (ii) the request to access contention slot is lost due to collision and the retransmitted requests fall out of the NACKDelayTimer for the current collection period. The calculation of NACKDelayTimer and StatusRetryTimer are as below:

$$
\begin{aligned}
& \text { NACKDelayTimer }=\frac{1}{2} \frac{L \cdot D}{C_{f}} \\
& \text { StatusRetryTimer }=N A C K \text { DelayTimer }+t_{R T T}
\end{aligned}
$$

where $L$ is number of FEC blocks per segment, $D$ is number of segments per MFTP block and $C_{f}$ is forward link capacity and $t_{R T T}$ is round-trip-time delay.

To compare the two policies, we simulate $N=\{50,100,150$, $200\}$ number of active receivers using $10 \%$ of contention slots in every NACKDelayTimer. If a request to access a contention slot collides with another request to the same slot, then the receivers randomly access another slot. In each attempt, a new CSI are readily available, which is calculated every $t_{\text {updats }}$ (seconds). At the receivers' terminal, after the last segment of a multicast block is received, the receivers respond to StatusRequest by sending NACK messages if there exists at least one segment in the multicast block received in error. In MFTP + CSI, each NACK message triggers access to a contention slot. The offered load on the contention channel that is due to NACK messages is:

$$
\rho_{N A C K}=\frac{N_{N A C K}}{\text { NACKDelayTimer }}=\frac{N_{N A C K}}{\frac{1}{2} \frac{L \cdot D}{C_{f}}}=\frac{N_{N A C K}}{L \cdot D} \cdot 2 C_{f}
$$

The loading on the contention channel can also be normalized to the amount of uplink capacity designated for contention access, $C_{C S}$ :

Contention channel loading $=\frac{\rho_{N A C K}}{C_{C S}}=\frac{N_{N A C K}}{L \cdot D} \cdot \frac{2 C_{f}}{C_{C S}}$

In Figs 2(a)-(c), we observe the performance of the two policies with the following simulation parameters: $L=1 \mathrm{FEC}$ block per segment with size 1000 bits, $D=1000$ segments per block, $C_{f}=100 \mathrm{kbps}$, frame duration is $80 \mathrm{~ms}$, there are 4 contentions slots in one frame duration and $C_{C S}=25$ slots. In Fig. 2(a), the contention channel loading for MFTP+CSI is plotted against number of active receivers $N$. It is observed that the collision rate increases with $N$ due to higher number of receivers with NACKs trying to access a fixed number of 
contention slots. Then in Fig. 2(b) and (c), we compare the two policies: FIS+CAS and MFTP+CSI. From the figure, in a system with a low number of active receivers, FIS+CAS perform better since resource utilization is higher due to timely CSI updates. However at large number of receivers, i.e. at $\mathrm{N}=200, \mathrm{MFTP}+\mathrm{CSI}$ performs better. The same scenario is observed in Fig. 2(c), FIS+CAS performs better at low number of active receivers and MFTP+CSI performs almost on par with FIS+CAS at large number of receivers. This is because in a small number of receivers, FIS+CAS manage to exploit a reliable set of CSI values from the timely update. The CAS functionality is optimized at this point, even with a fixed number of available uplink slots, $S_{n}$. However, as the size of multicast group increases, the error between updated CSI at the $\mathrm{RNC}$, and the CSI measured at the receivers' terminal when data is received, is large for a fixed $S_{n}$.

In contrast, in MFTP+CSI the scheduler calculates $\hat{P}_{R t x}$ at the end of StatusRetryTimer which is set to $5.8 \mathrm{~s}$. A user that updates its CSI values at the beginning of the StatusRetryTimer might have a change of CSI values by the time the RNC calculates $\hat{P}_{R t x}$. Hence, in this scenario the scheduler decides on samples that are already obsolete. Furthermore, it may occur instances when NACK messages arrive later than the corresponding StatusRetryTimer due to collisions. Thus a late NACK message can only be considered in the next pass. A possible solution to this scenario is to increase StatusRetryTimer to include late NACKs. However, obviously it also increases the delay between passes.

Even with all peculiarities in MFTP+CSI due to random access mechanism, the approach performs better in a large multicast group, because in random access mechanism a collided feedback message can still be updated using retransmission mechanism. In essence, $\mathrm{MFTP}+\mathrm{CSI}$ policy is suitable for large multicast group size whereas FIS +CAS policy is more practical for a system with a smaller number of receivers. However, if FIS+CAS deploy a larger value of $S_{n}$, its performance might be good even at large multicast group size.

\section{CONCLUSION}

In this paper, we propose a feedback implosion suppression algorithm that reduces the volume of feedback information amongst receivers in a multicast group in order to select and assign receivers over a limited number of available uplink slots. It complements the CSI collection policy, which collects feedback from active receivers to track down changes of channel levels. The proposal is compared with a random access mechanism that suits the above transport protocol.

In conclusion, FIS +CAS manage to avoid collision problem and delays due to random access timers by assigning fixed available uplink slots to selected receivers. Also, in FIS +CAS NACK update is treated as a parameter to select receivers rather than as a determinant for uplink access. In other words, NACK semantics are violated and this mechanism is not suitable for MFTP that runs on transport layer to ensure reliable transmission.
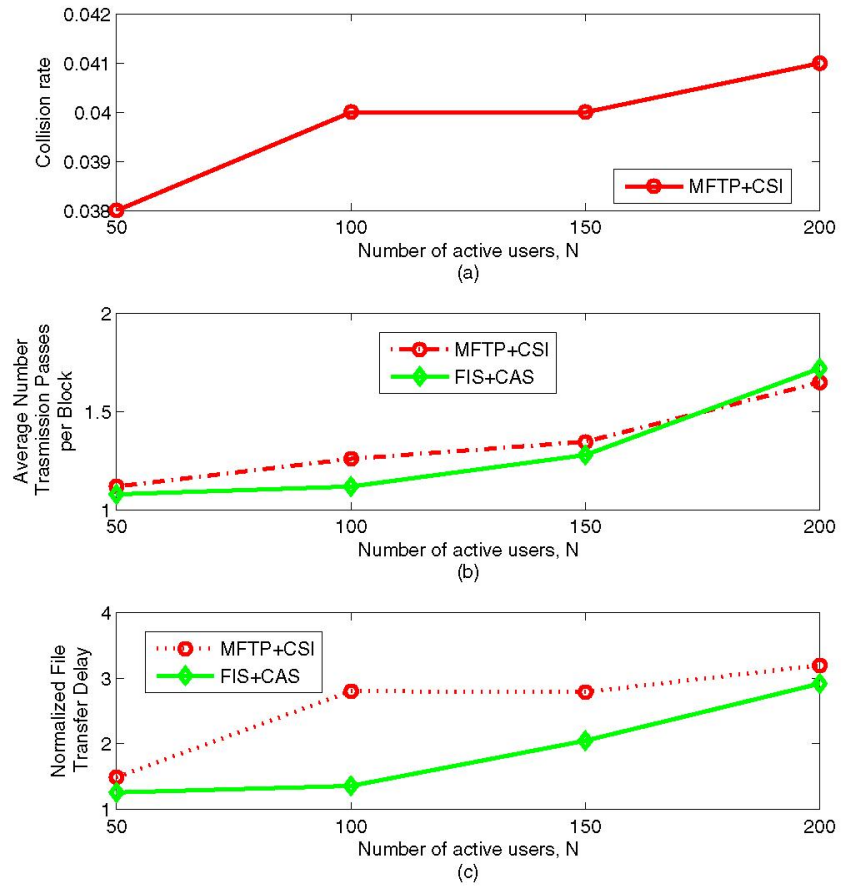

Fig. 2 (a) Collision rate vs number of active receivers, (b) Average number of transmission passes per block vs number of active receivers, (c) Normalized file transfer delay vs number of active receivers. For FIS $+\mathrm{CAS}$, $S_{n}=20$ slots; for MFTP $+\mathrm{CSI}, C_{C S}=25$ slots

Alternatively, in implementing CSI updates using a random access mechanism to suit the MAC and transport protocols that run in parallel for reliable transmission, several main concerns should be addressed. They are: ( $i$ ) long session delay due to StatusRequestTimer. One retransmission pass adds one StatusRequestTimer and one round-trip-time delay, (ii) the CAS functionality could be sub-optimal because the CSI updates are collected over a long duration (one StatusRequestTimer) and the CSI values could be obsolete by the time the StatusRequestTimer ends and scheduling decision has to be executed. For both constraints, the solution can be achieved by adapting the size of a multicast block, hence a shorter span of StatusRequestTimer, which could lead to shorter session delay and more timely scheduling decision.

Hence, for further investigation it is proposed to have an adaptive downlink transmission where the size of multicast block is determined depending on the reported CSI. A shorter block size gives low normalized file transfer delay but large contention channel loading which can lead to a collision problem. In essence, it is an optimization problem where it is intended to set the block size according to $P_{R t x}$ such that both session delay and contention channel loading are minimized.

\section{REFERENCES}

[1] A.Sali, G.A., B.G.Evans. A Cross-layer Approach for Packet Scheduling in Reliable Multicast Data Transmission over Geostationary Satellite Networks. in Vehicular Technology Conference - Spring 2007. 2007. Dublin. 
2. G. Akkor, M.H., J.S. Baras. A Multiple Subset Sum Formulation for Feedback Implosion Suppression over Satellite Networks. in IEEE GLOBECOM. 2005.

3. I.Alocci,M.B.,N.Celandroni,G.Giambene,S.Karapantazis. Proposal of a Reliable Multicast Protocol in a HAP-Satellite Architecture. in Vehicular Technology Conference-Spring. 2007. Dublin.

4. L.E.Braten, T.T., Semi-Markov Multistate Modeling of Land Mobile Propagation Channel for Geostationary Satellites. IEEE Transactions on Antennas and Propagation, December 2002. 50(12).

5. K.Miller, K.R., StarBurst Multicast File Transfer protocol (MFTP) Specification. IETF Draft<draft-miller-mftp-spec-03.txt>, July 1998.

6. S. Martello, P.T., Knapsack Problems Algorithms and Computer Implementations, J.W.S. Ltd, Editor. 1990. 